\title{
IMPLEMENTAÇÃO DE FERRAMENTAS DE ENGENHARIA DE PRODUÇÃO: O IMPACTO EM UM PROGRAMA DE EDUCAÇÃO TUTORIAL
}

DOI: 10.37702/2175-957X.COBENGE.2021.3699

Victor da Silva Magalhães - victor.magalhaes@engenharia.ufjf.br Universidade Federal de Juiz de Fora

Rua orestes homem da costa 189

28470-000 - Santo Antônio de Pádua - RJ

Lucimar Bonfá Coelho de Oliveira - lucimar.oliveira@engenharia.ufjf.br Universidade Federal de Juiz de Fora

Avenida dos Andradas 553

36036-000 - Juiz de Fora - MG

Igor Augusto Delvivo - igor.delvivo@engenharia.ufjf.br

Universidade Federal de Juiz de Fora UFJF

Rua Elvira Bellei 673

36030-560 - Juiz de Fora - MG

Juliana Veiga de Souza Frango - juliana.frango@engenharia.ufjf.br

Universidade Federal de Juiz de Fora

Rua Halfed 1097

36016-000 - Juiz de Fora - MG

Roberta Cavalcanti Pereira Nunes - rcpnunes@gmail.com

Universidade Federal de Juiz de Fora

Rua Oscavo Gonzaga Prata 372

36033-220 - Juiz de Fora - MG

Resumo: Diante de um cenário no qual a capacitação contínua e uma gestão excelente são essenciais para o alcance de resultados, os membros do Grupo de Educação Tutorial do curso de Engenharia de Produção da Universidade Federal de Juiz de Fora (UFJF), optaram por descentralizar os aprendizados do seu curso para extendê-los para outras organizações estudantis, e assim contribuir para o desenvolvimento das mesmas. Portanto, o objetivo deste artigo é apresentar como 
a implementação de três oficinas dos temas de engenharia de produção - Layout, Planejamento Estratégico e 5s - impactaram positivamente em um Programa de Educação Tutorial da Engenharia Elétrica da UFJF. A pesquisa-ação foi usada como metodologia do estudo devido a ação direta dos pesquisadores com os participantes e a resolução dos problemas.

Palavras-chave: Planejamento Estratégico, Layout, 5s 


\section{IMPLEMENTAÇÃO DE FERRAMENTAS DE ENGENHARIA DE PRODUÇÃO: O IMPACTO EM UM PROGRAMA DE EDUCAÇÃO TUTORIAL}

\section{INTRODUÇÃO}

Baseados em ensino, pesquisa e extensão, os grupos GETs (Grupo de Educação Tutorial) e PETs (Programa de Educação Tutorial) objetivam "apoiar grupos de alunos que demonstrem potencial, interesses e habilidades destacadas em cursos de graduação das Instituições de Ensino Superior - IES" (MOB, 2006, p.4). Assim sendo, a melhoria na formação acadêmica dos integrantes se alinha com os objetivos básicos do grupo como a formação acadêmica ampla, a interdisciplinaridade e a atuação coletiva.

Dito isso, os alunos que fazem parte do GET- Produção da Universidade Federal de Juiz de Fora estudam áreas específicas do curso de engenharia de produção, tais como Planejamento Estratégico, SCRUM, Metodologia 5S, Layout, entre outros, vitais para o gerenciamento interno e funcionamento efetivo tanto de qualquer empresa quanto de outros grupos e segmentos da UFJF.

O Grupo de Educação Tutorial do curso de Engenharia de Produção (GET-Produção) da Universidade Federal de Juiz de Fora (UFJF), alinhando tais objetivos, traçou e aplicou melhorias julgadas essenciais para que o trabalho coletivo, do Programa de Educação Tutorial do curso de Engenharia Elétrica (PET-Elétrica), alcançasse uma maior produtividade e alinhamento de equipe, que se encontravam insatisfeitos com o rendimento, direcionamento e organização do grupo. Assim, o objetivo deste trabalho é apresentar o impacto que as ferramentas de engenharia de produção, Planejamento Estratégico (PE), 5S e Layout, promoveram ao ser aplicadas dentro do grupo PET - Elétrica da UFJF.

Tendo em vista que o funcionamento atual da grande parte dos Grupos e Programas de Educação Tutorial (GETs e PETs) e outros segmentos da engenharia seguem um modelo empresarial, a aplicação de tais ferramentas organizacionais como Layout, onde é feito um rearranjo do espaço físico disponível para melhor aproveitamento e produtividade, e Metodologia 5S, em que são criados sugestões de hábitos e organizações para que as tarefas desenvolvidas por determinadas pessoas seja mais fluida e ágil, é fundamental para a plena atividade de tais grupos e suas respectivas equipes.

O corpo deste artigo está dividido em 7 seções. Na seção 1, Introdução, é apresentada uma visão geral sobre o trabalho; na seção 2, Referencial Teórico, são expostos os principais assuntos abordados neste artigo: Programas de Educação Tutorial, planejamento estratégico, 5S e Layout; na seção 3, Metodologia, é apresentada a metodologia utilizada para a realização do trabalho; na seção 4, o desenvolvimento das aplicações e na seção 5, como foi a validação dessas aplicações; já na seção 6 são apresentados os Resultados e Discussões; e por fim na seção 7, a conclusão do estudo.

\section{REFERENCIAL TEÓRICO}

\subsection{Grupos ou Programas de Educação Tutorial e suas relações}

O Programa de Educação Tutorial (PET), do qual o grupo PET-Elétrica UFJF está inserido, surgiu em 1979 com o objetivo de buscar os melhores candidatos e oferecer-lhes 
as condições de crescimento intelectual. Segundo o Portal do Ministério da Educação existem 842 grupos distribuídos em 121 IES (Instituições de Ensino Superior).

Como ramificação desse programa surgiram os Grupos de Educação Tutorial (GET) ou PET Institucional que são gerenciados pelas próprias IES. No Art. I da Resolução No 69/2008 a Universidade Federal de Juiz de Fora criou os GETs, nos moldes do Programa Nacional PET. Assim, a UFJF hoje contém 6 grupos PET e 8 grupos GET. Esses grupos interagem com troca de experiências e conhecimentos específicos, para assim, se auxiliarem na realização de suas atividades. Sendo assim, a troca mútua de conhecimentos tange oportunidades reais de aprendizados, networking, ampliação de horizontes e contribuição para o desenvolvimento pessoal e profissional dos envolvidos no processo.

Nesse cenário, o PET-Elétrica solicitou ao GET-Produção por conhecimentos específicos da área de Engenharia de Produção que pudessem auxiliar em aumentar a produtividade e alinhar os objetivos do grupo. E desta forma, o GET-Produção optou por oferecer os treinamentos em Layout, Metodologia 5s e Planejamento Estratégico ao PET-Elétrica a fim de sanar sua demanda.

\subsection{Arranjo físico ou Layout}

Segundo Moreira (2012), o arranjo físico, também conhecido por layout, define o posicionamento das instalações, mesas, máquinas, equipamentos e pessoas da operação. Ademais, inspeciona a segurança, atratividade, flexibilidade e eficiência de uma operação e determina o modo pelo qual os recursos fluem pela operação. Para Neumann e Scalice (2015), os espaços de trabalho começaram a ser utilizados intuitivamente, porém desde o desenvolvimento dos novos sistemas produtivos em meados do século $\mathrm{XX}$, e com as novas exigências do mercado globalizado, houve a necessidade de dar uma atenção maior a distribuição e ao arranjo dos setores de trabalho, fazendo com que o layout assumisse um papel importante no processo produtivo.

Segundo Neumann e Scalice (2015), um layout eficiente, do ponto de vista econômico, contribui para a redução dos custos de produção. Além disso, a reorganização do layout deve ser uma atividade constante em qualquer organização que tenha como prioridade a eficiência em sua área de atuação, visto que há uma evolução tecnológica constante, tornando modelos e métodos obsoletos.

Ainda para Neumann e Scalice (2015), os tipos básicos de layout definem o sistema de organização da produção, variando com a natureza dos produtos e tipos de operações. A tabela 1, apresenta as definições dos tipos de arranjo físico mais comuns:

Tabela 1: Definições dos tipos de arranjos físicos mais comuns.

\begin{tabular}{|l|l|}
\hline Layout por produto ou linear & $\begin{array}{l}\text { De acordo com Jones e George (2008), no layout por } \\
\text { produto o trabalhador fica parado e o produto é movido } \\
\text { até as estações de trabalho para ser montado. Segundo } \\
\text { Neumann e Scalice (2015) ele é usado quando um } \\
\text { produto é fabricado em grande quantidade, e para isso, } \\
\text { as máquinas ou estações de trabalho são organizadas } \\
\text { de acordo com a sequência de montagem do produto, a } \\
\text { fim de obter um fluxo linear. }\end{array}$ \\
\hline
\end{tabular}




\begin{tabular}{|c|l|}
\hline Layout por processo ou funcional & $\begin{array}{l}\text { Segundo Neumann e Scalice (2015) no layout por } \\
\text { processos, as máquinas são agrupadas de acordo com } \\
\text { as funções que desempenham. Esse tipo de layout, tem } \\
\text { a característica de formar setores que são } \\
\text { especializados em determinadas tarefas, onde as } \\
\text { máquinas e equipamentos ficam fixos e o produto se } \\
\text { movimenta. }\end{array}$ \\
\hline Layout celular & $\begin{array}{l}\text { De acordo com Slack, Brandon-Jones e Johnston } \\
\text { (2018), em um arranjo físico celular, os recursos são } \\
\text { processados em uma parte da operação (ou célula) e } \\
\text { após transformados, seguem para a próxima. Além } \\
\text { disso, cada célula pode ser organizada em um layout } \\
\text { funcional ou em linha. }\end{array}$ \\
\hline Layout posicional ou fixo & $\begin{array}{l}\text { Segundo Slack, Brandon-Jones e Johnston (2018), no } \\
\text { arranjo físico posicional quem sofre o processamento } \\
\text { fica em um local fixo, enquanto os equipamentos, } \\
\text { maquinário, instalações e pessoas se movem conforme } \\
\text { necessário. Isso acontece quando o produto é muito } \\
\text { grande ou delicado, por exemplo. }\end{array}$ \\
\hline Layout Misto & $\begin{array}{l}\text { Para Neumann e Scalice (2015), os layouts mistos, } \\
\text { também conhecidos como híbridos, resultam da } \\
\text { utilização de mais de um tipo de layout em uma mesma } \\
\text { unidade de produção, devido ao alto volume de } \\
\text { produção. Isso ocorre porque as empresas têm que se } \\
\text { adaptar às demandas do mercado e para atendê-las da } \\
\text { melhor maneira, apenas um tipo de arranjo físico não é } \\
\text { suficiente, mas sim uma combinação de alguns deles. }\end{array}$ \\
\hline
\end{tabular}

Fonte: Os autores (2021).

Assim como qualquer empresa, as organizações estudantis precisam de um bom layout físico, visto que, segundo Ching et al. (2019), um bom layout pode contribuir para a produtividade, reduzindo custos operacionais e desperdícios.

\subsection{Metodologia $5 \mathrm{~s}$}

Para Xenos (1998), o termo 5s é a derivação de cinco palavras japonesas, são elas: Seiri, Seiton, Seisou, Seiketsu e Shitsuke, traduzidas para a língua portuguesa como senso de utilização, ordenação, limpeza, saúde e autodisciplina. Segundo Colenghi (2013) e Carvalho (2011), o programa 5S é uma metodologia da Gestão da Qualidade Total baseada em 5 princípios (5 sensos): o Seiri, senso que trata da utilidade e do descarte de itens não utilizados em uma organização; o Seiton, refere-se a ordenação do espaço físico visando agilidade e facilidade de acesso aos recursos; o Seiso, atua na limpeza do ambiente e a redução de desperdícios; o Seiketsu, o senso do bem-estar no ambiente, isto é, as condições propícias à saúde física e mental; e o Shitsuke, referente a autodisciplina e o compromisso com a manutenção do programa $5 \mathrm{~S}$ e dos outros sensos.

Correia et al. (2010) citam como vantagens do 5S: a melhoria do ambiente de trabalho, da saúde e da segurança; a diminuição de movimentos desnecessários; e a liberação de espaços; os quais são essenciais para a instalação do método quando realizadas de maneira consciente e em cooperação com os funcionários.

A implantação desta metodologia pretende organizar e estabelecer novos projetos de melhoria contínua, permitindo que o PET-Elétrica aprimore seu desenvolvimento, 
reduza os desperdícios e propicie um ambiente para melhor desempenho na qualidade de vida de seus membros.

\subsection{Planejamento Estratégico}

\section{Conceito}

O Planejamento Estratégico é uma das ferramentas administrativas mais importante da atualidade, através dele o gestor e toda a equipe definem o objetivo maior da instituição e criam sua identidade. Por meio desse objetivo são traçados os caminhos e os meios para que o mesmo seja realizado. De acordo com Philip KOTLER (1975), o Planejamento Estratégico é uma metodologia gerencial que permite estabelecer a direção a ser seguida pela Organização, visando maior grau de interação com o ambiente.

Através do planejamento estratégico, a corporação espera: conhecer e utilizar melhor seus pontos fortes; entender e eliminar ou adaptar seus pontos fracos; reconhecer e aproveitar as oportunidades; identificar e evitar as ameaças; e obter um plano de trabalho efetivo (OLIVEIRA, 2018). Por conseguinte, antes de fazer o planejamento estratégico a organização precisa definir a si mesma (CRUZ, 2017). Segundo Maximiano (2011, p.62), é preciso seguir os seguintes passos para estabelecer a estratégia do grupo: "Onde estamos agora? > ponto de partida, qual caminho percorrer? Quais as etapas intermediárias? Onde queremos chegar? > Objetivo".

\section{Análise Ambiental}

Então, deve-se realizar a análise ambiental da instituição a fim de estabelecer a estratégia da mesma, pois segundo os autores Certo e Peter (2005), a análise ambiental é o conjunto de todos os fatores, tanto internos quanto externos, que podem influenciar o progresso obtido por meio da realização dos objetivos.

Como Barbalho (1997) bem define, a análise da organização é dividida sob dois aspectos: a análise externa, que pretende identificar as ameaças e as oportunidades do mercado e o melhor modo de lidar com elas; e a análise interna, que pretende determinar os pontos fortes e fracos do próprio grupo e como eles podem ser potencializados e amenizados, respectivamente.

\section{Análise SWOT}

Com as informações obtidas na análise ambiental da instituição pode-se realizar então a análise SWOT, que é uma ferramenta com a finalidade de avaliar os ambientes internos e externos, formulando estratégias de negócios para a instituição. De acordo com Cruz (2017), a Análise SWOT é considerada uma sigla formada pelas palavras: Strengths (pontos positivos), Weaknesses (fraquezas), Opportunities (oportunidades) e Threats (ameaças). Por meio dessa ferramenta, é possível analisar os pontos fortes e fracos da empresa. Segundo Andrade (2016), a Análise SWOT é dividida em duas partes. A primeira, é denominada fatores internos e tem como finalidade identificar os diferenciais e as vantagens competitivas que a empresa pode explorar. A segunda, intitulada como fatores externos, consiste em identificar as ameaças e oportunidades decorrentes do relacionamento entre a organização e as condições ambientais.

O propósito da Análise S.W.O.T. é identificar o maior número possível de forças, fraquezas, oportunidades e ameaças, de forma que ao ser realizado o cruzamento das informações se possa traçar um planejamento de objetivos e ações de acordo com a realidade encontrada. 


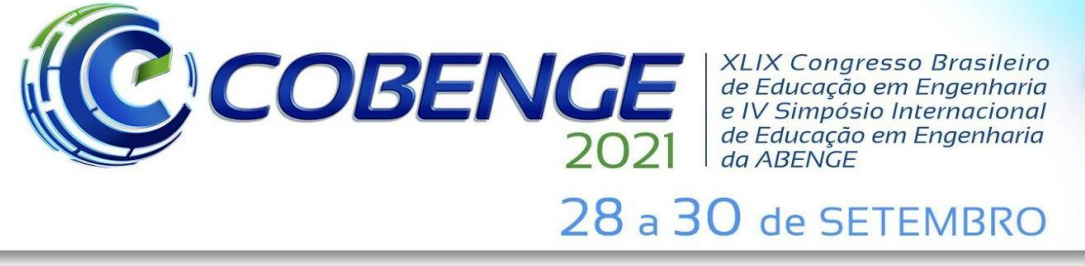

\section{METODOLOGIA}

Este estudo classifica-se como uma pesquisa aplicada, quanto à natureza; descritiva, quanto aos objetivos; e qualitativa, quanto à forma de abordagem. Segundo Quitério (2010), uma pesquisa é aplicada quando o objetivo é gerar conhecimentos a fim de solucionar problemas específicos por meio de aplicação prática; descritiva quando visa retratar determinado fenômeno, envolvendo o uso de técnica como questionários; e qualitativa quando o ambiente natural se torna fonte para coleta de informações. $\mathrm{O}$ uso desses métodos é justificado pelo seguinte motivo: a partir do questionário, de caráter qualitativo, contendo perguntas de múltipla escolha e com respostas escalonadas, foi possível obter informações, acerca do impacto das ferramentas de Engenharia de Produção usadas nas aplicações práticas no PET-Elétrica, que foram analisadas posteriormente.

Além disso, o trabalho pode ser apresentado como uma pesquisa-ação, visto que através do desenvolvimento das oficinas de Layout, $5 \mathrm{~S}$ e PE no PET-Elétrica - que auxiliaram o grupo na elaboração de soluções para sanar problemas reais nos quais está inserido - foi possível realizar a pesquisa necessária para elaborar as análises do impacto das ferramentas. Para Thiollent (1985), essa metodologia é um tipo de pesquisa social que é planejada e realizada em associação próxima com uma ação ou com a resolução de um problema coletivo, em que os pesquisadores e os participantes representativos da situação real investigada estão envolvidos de modo cooperativo e participativo.

\section{DESENVOLVIMENTO DAS APLICAÇÕES}

Neste tópico, apresentam-se os meios pelos quais foram desenvolvidas as aplicações das oficinas que conduziram a preparação deste artigo. Ele está estruturado em Oficinas de layout, PE e 5S e Coleta de dados.

\subsection{Oficinas de Layout, $5 S$ e PE}

\section{Layout}

Inicialmente, os membros do projeto foram até a instalação física do PET-Elétrica e registraram o ambiente através de fotos. As imagens foram analisadas pelos integrantes, que criaram uma folha com a planta baixa, e outros papéis menores representando os móveis da sala. Nessa etapa, foi estudado também qual dos tipos de layout seria o mais adequado.

Após, no dia marcado, o GET-Produção apresentou por meio de slides o conhecimento sobre layout, seus princípios e tipos, e como escolher o melhor tipo. Em seguida, os membros do PET-Elétrica montaram com os papéis disponibilizados e de acordo com o que foi exposto na teoria, um novo arranjo ideal com auxílio dos ministrantes. Ao término dessa etapa, foi realizada uma roda de conversa com ênfase nas dificuldades encontradas na montagem, além do compartilhamento do resultado obtido e da experiência dos envolvidos, finalizando duas horas de oficina. A mudança física para o modelo estabelecido foi executada pelo PET-Elétrica duas semanas depois.

\section{Metodologia 5S}

De maneira análoga ao método utilizado no Layout, o grupo foi à sala do PET-Elétrica para a retirada de amostras, através de fotos, da organização do local antes da aplicação da oficina. As mesmas foram usadas posteriormente na apresentação de 
slides do treinamento, com o objetivo de incentivar o senso crítico e o aprendizado sobre a ferramenta aos membros.

No dia estabelecido para a oficina, foram expostos os conhecimentos sobre a origem da ferramenta, sobre os cinco sensos (Seiri, Seiton, Seiso, Seiketsu e Shitsuke) e dicas sobre como manter as alterações feitas através do programa. Em seguida, houve um espaço para a discussão das possíveis mudanças a serem realizadas na sala. Assim, os membros escreveram, a partir do aprendizado, uma lista de afazeres para a melhoria e manutenção da organização do local. A oficina durou por volta de 2 horas, e as melhorias foram implementadas no mesmo dia da modificação do layout da sala.

\section{Planejamento Estratégico (PE)}

Assim como as outras oficinas, o PE também precisou de um levantamento dos dados antes, com o intuito de averiguar se o PET-Elétrica possuía algum tipo de planejamento, estabelecimento de objetivos ou metas. Logo após, com uma série de orientações a serem seguidas mediante uma apresentação de slides, foram estabelecidos três dias para a conclusão do PE, durando 2 horas cada.

No primeiro dia expõe-se a definição do que é o planejamento estratégico. Em seguida, os membros fizeram a análise ambiental, definindo o público interno e externo do PET-Elétrica, assim como pontos fracos e fortes, e ameaças e oportunidades do grupo. A partir desses tópicos, foi elaborada a Matriz SWOT Cruzada, utilizando-se do cruzamento das análises feitas anteriormente. Por conseguinte, no segundo dia, foram criadas a missão, visão, valores e negócio da equipe. Por fim, já no terceiro dia, definiu-se os objetivos estratégicos, as metas, os indicadores e os planos de ação que serão cumpridos ao longo do ano de atividades do PET. Todas as decisões tomadas foram desenvolvidas por meio de dinâmicas, baseadas na técnica de brainstorming, depois da apresentação da base teórica.

\subsection{Coleta de dados}

Durante a aplicação das oficinas citadas, os membros do GET-Produção orientaram os participantes a fim de ajudá-los a sanar dúvidas e propor ações, atuando como facilitadores. Essa técnica pode ser chamada de observação participante, que segundo Gil (2008), consiste na participação real do pesquisador na vida da comunidade, da organização ou do grupo em que é realizada a pesquisa.

Outro método utilizado para a coleta de dados foi o questionário aplicado logo após o término da realização dessas oficinas, com o intuito de recolher feedbacks acerca da qualidade dos treinamentos e sugestões para o aprimoramento dos mesmos. De acordo com Gil (2008), o questionário pode ser definido "como a técnica de investigação composta por um número mais ou menos elevado de questões apresentadas por escrito às pessoas, tendo por objetivo o conhecimento de opiniões, crenças, sentimentos, interesses, expectativas, situações vivenciadas etc.".

Também, seis meses após a aplicação das oficinas no PET-Elétrica, um novo questionário de feedback foi disponibilizado para os participantes com o intuito de constatar se as ferramentas 5S, Layout e PE trouxeram melhorias para o segmento. 


\section{VALIDAÇÃO DAS APLICAÇÕES}

\subsection{Questionário 1 - ao fim das aplicações}

A partir dos dados obtidos nessa pesquisa, foram analisadas as respostas de 13 membros do PET-Elétrica, sendo $81,2 \%$ do total de participantes do grupo. Destes, 11 $(84,6 \%)$ estiveram presentes na oficina de $5 \mathrm{~S}$ e 12 (92,3\%) nas de PE e Layout.

Conforme Reichheld (2006), o Net Promoter Score (NPS) é uma métrica de desempenho gerada a partir de uma só pergunta: "Você indicaria nossa empresa para um amigo ou conhecido?". Contudo, ela pode ser adaptada para as mais diversas situações como no nosso caso, em que foi feito o questionamento: "O quanto você recomendaria este minicurso para um amigo?". Dessa forma, podemos avaliar a satisfação dos participantes em relação às oficinas oferecidas. Ainda, de acordo com a ferramenta NPS, é possível classificar as empresas em quatro zonas, sendo a de excelência, de maior grau, no qual o NPS encontra-se na faixa de 75 a 100. Visto isso, pode-se constatar que o serviço oferecido ao PET-Elétrica gerou uma experiência positiva aos partícipes, pois os NPSs registrados foram 77, 86 e 93 para o PE, 5S e Layout, respectivamente.

\subsection{Questionário 2 - seis meses após às aplicações}

Com base nas informações adquiridas no segundo questionário de feedback, foi elaborada a Tabela 2, a qual expõe quais foram os três fatores mais relevantes para o grupo com o uso de cada ferramenta, de acordo com os votos dos participantes.

Tabela 2 - Importância de cada ferramenta de acordo com os quesitos mais votados.

\begin{tabular}{|c|c|c|c|c|c|}
\hline \multicolumn{2}{|c|}{ Planejamento Estratégico } & \multicolumn{2}{c|}{ Layout } & \multicolumn{2}{c|}{5 Sotos } \\
Quesitos & (un) & Quesitos & $\begin{array}{c}\text { Votos } \\
\text { (un) }\end{array}$ & Quesitos & $\begin{array}{c}\text { Votos } \\
\text { (un) }\end{array}$ \\
\hline $\begin{array}{c}\text { Definiu o foco de } \\
\text { atuação do grupo }\end{array}$ & 12 & $\begin{array}{c}\text { Utilizou o espaço } \\
\text { existente da forma } \\
\text { mais eficaz possível }\end{array}$ & 10 & $\begin{array}{c}\text { Manteve o local de } \\
\text { trabalho } \\
\text { organizado }\end{array}$ & 12 \\
\hline $\begin{array}{c}\text { Possibilitou o grupo a } \\
\text { fazer um diagnóstico } \\
\text { de oportunidades e } \\
\text { melhorias }\end{array}$ & 10 & $\begin{array}{c}\text { Proporcionou um } \\
\text { fluxo de } \\
\text { comunicações e de } \\
\text { trabalho mais } \\
\text { eficiente }\end{array}$ & 8 & $\begin{array}{c}\text { Ajudou a manter o } \\
\text { ambiente limpo }\end{array}$ & 10 \\
\hline $\begin{array}{c}\text { Auxiliou na tomada de } \\
\text { decisão }\end{array}$ & 8 & $\begin{array}{c}\text { Proporcionou um } \\
\text { clima mais favorável } \\
\text { para o trabalho }\end{array}$ & 8 & $\begin{array}{c}\text { Auxiliou na } \\
\text { procura de } \\
\text { materiais }\end{array}$ & 9 \\
\hline
\end{tabular}

Fonte: Os autores (2021).

Na pesquisa também foi examinado se, de fato, a implementação dos minicursos está sendo enriquecedora ao grupo PET-Elétrica, beneficiando-o no que diz respeito aos objetivos do mesmo. Para esse fim, observamos as respostas obtidas no questionário baseadas na pergunta: "Você acha que a oficina X está sendo válida ao grupo?", sendo X o nome de cada uma das oficinas aplicadas. Os dados demonstram que mais de $72 \%$ dos 
participantes da aplicação de $5 \mathrm{~S}$ e Layout consideram os treinamentos válidos, e mais de 90\% dos partícipes de PE concordam com a mesma afirmação, como mostra a Figura 1.

Figura 1 - Porcentagem de participantes que consideram as oficinas válidas ao PET-Elétrica.
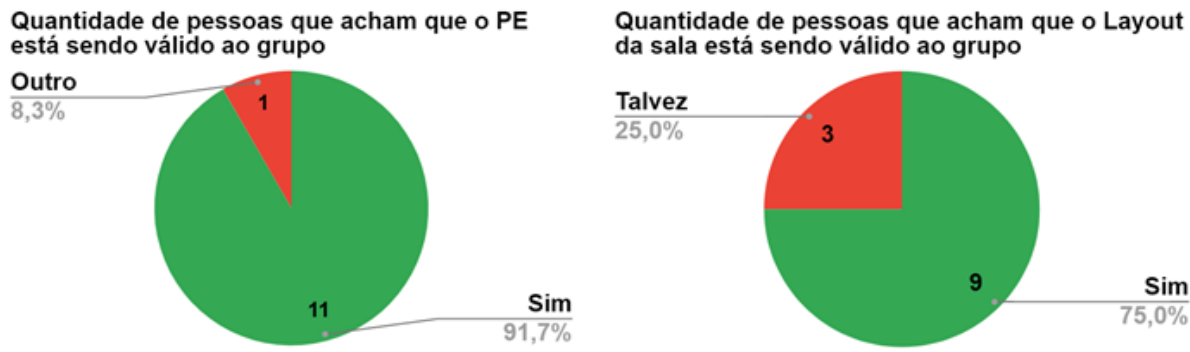

Quantidade de pessoas que acham que o 5 S está sendo válido ao grupo

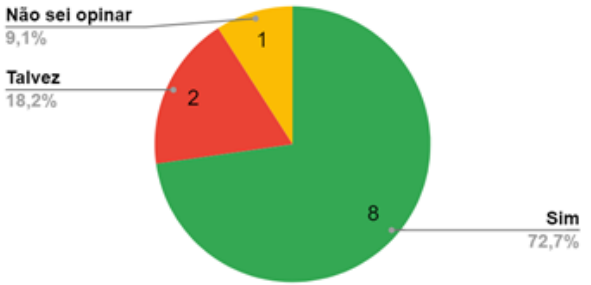

Fonte: Os autores (2021).

\section{RESULTADOS E DISCUSSÕES}

Nesta seção, serão abordados os resultados do projeto em sua totalidade, considerando as mudanças ocorridas no PET-Elétrica com o desenvolvimento das aplicações, assim como as informações de validação obtidas no tópico anterior.

As alterações executadas acerca da oficina de Layout, inicialmente, efetuaram-se a partir da escolha do layout celular, visto que temos várias atividades diferentes sendo desenvolvidas dentro da sala do grupo. Após, cada célula foi organizada em layout linear visto que os materiais a serem utilizados estão dispostos de acordo com a sequência de trabalho, levando a um fluxo contínuo. Diante disso, concluímos que o tipo de layout aplicado no PET-Elétrica foi o layout misto, uma vez que foi utilizado mais de um tipo de arranjo físico. O novo layout proporcionou uma melhor organização do espaço físico disponível, além de possibilitar um melhor fluxo de comunicações e trabalho eficiente, levando a um clima mais favorável para o trabalho, sendo essas afirmações apuradas por meio do segundo questionário adotado.

As mudanças proporcionadas pela aplicação da metodologia $5 S$ puderam solucionar problemas tais como mochilas dispostas em cima das mesas, materiais misturados e fios desorganizados. Essas modificações, assim como as anteriormente citadas, proporcionaram uma melhor organização do ambiente de trabalho, além de ajudar na manutenção da limpeza do local e facilitar a busca por materiais, de acordo com os dados obtidos através do questionário 2.

Posto que o PET-Elétrica não possuía nenhum tipo de planejamento que direcionava o grupo, a oficina de Planejamento Estratégico cumpriu seu objetivo de auxiliar nessa construção, uma vez que foram definidos os objetivos da organização, como "fortalecer a marca e os projetos". Ademais, a missão ficou acordada como: "Desenvolver atividades acadêmicas capazes de complementar a formação técnica e humana dos discentes, a fim de formar engenheiros eletricistas que transformem a sociedade"; os valores necessários aos membros do grupo, sendo: "Comprometimento; 
Espírito de Equipe; Proatividade; Liderança; Pontualidade; Criatividade; Motivação; Senso crítico; Organização". e o negócio foi acertado como: "Inspirar disseminando conhecimentos e experiências".

Dentre as limitações encontradas na pesquisa, podem ser citadas a não participação de três membros do PET-Elétrica nos questionários aplicados, e, também, devido a pandemia do novo coronavírus não pudemos ter acesso a sala do grupo para deixar o estudo com um maior nível de precisão no que se refere a efetividade e uso do novo layout, e da manutenção e cumprimento da metodologia 5 S.

\section{CONSIDERAÇÕES FINAIS}

O presente estudo buscou analisar o impacto das ferramentas de engenharia de produção - Planejamento estratégico (PE), 5S e Layout - no Programa de Educação Tutorial de Engenharia Elétrica da UFJF. Com base nos dados obtidos, apresentados na seção Resultados e Discussões, e através dos questionários aplicados no PET-Elétrica UFJF, os resultados atingidos meses após a execução dos projetos mostram a importância de um direcionamento sobre as ações do grupo, visando o alcance dos seus objetivos. Para tanto, foi concluído que a utilização das metodologias é benéfica em todos os níveis de organizações, sejam elas estudantis ou empresariais, posto que a instalação das mesmas possibilita melhorias internas em diferentes âmbitos institucionais, como estrutura organizacional, instalações físicas e fluxo de informações e processos.

Em relação ao GET-Produção, é necessário ressaltar que o grupo encontra-se em processo de melhoria contínua a fim de aprimorar seus treinamentos oferecidos à comunidade acadêmica, em busca de contribuir na formação de melhores profissionais, bem como na facilitação da transmissão de conhecimento. Desse modo, em trabalhos futuros, o grupo pretende expandir a aplicação das práticas aqui mencionadas (PE, 5S e Layout) para os demais segmentos da UFJF, visto que juntas desempenharam um resultado satisfatório no grupo analisado.

\section{Agradecimentos}

Agradecemos o apoio dado pelo grupo GET-Produção durante o processo de elaboração do artigo e também à Universidade Federal de Juiz de Fora, por proporcionar essa oportunidade aos autores.

\section{REFERÊNCIAS}

ANDRADE, A. R. Planejamento Estratégico - Formulação, Implementação e Controle. $2^{a}$ edição. Grupo GEN, 2016.

CARVALHO, P. O programa 5S e a qualidade total. 5.a Ed. Campinas: Alínea, 2011.

CHING, H. Y; DUARTE, A; RIEG, D; SCRAMIM, F. Administração da produção e operações, uma abordagem inovadora com desafios práticos. 1a. Edição. Editora Empreende, 2019.

COLENGHI, V. O \& M e Qualidade Total: Uma integração perfeita. 2.a Ed. Rio de Janeiro: Qualitymark, 2013. 
CORREIA, B. R. B.; BARREIRA M. V. S.; FIGUEIREDO F. J. S.; BALTAZAR C. C. Implantação do Programa 5S em um setor de injeção termoplástica sob aspectos da melhoria contínua. In: Encontro Nacional de Engenharia de Produção, 30, 2010, São Carlos, Anais... São Carlos: Abepro, 2010.

CRUZ, T. Manual de Planejamento Estratégico. Grupo GEN, 2017.

GIL, Antonio Carlos. Métodos e técnicas de pesquisa social. 6. ed. São Paulo: Editora Atlas, 2008.

JONES, G. R.; GEORGE, J. M. Administração Contemporânea. Grupo A, 2008.

MINISTÉRIO DA EDUCAÇÃO. Manual de Orientações Básicas PET. Ministério da Educação, 2006. Disponível em: http://portal.mec.gov.br/pet/manual-de-orientacoes. Acesso em: 23 set. 2020.

MOREIRA, D. A. Administração da Produção e Operações - $2^{a}$ Edição Revista e Ampliada. Cengage Learning Brasil, 2012.

NEUMANN, C; SCALICE, R. K. Projeto de Fábrica e Layout. Grupo GEN, 2015.

OLIVEIRA, D. P. R. Planejamento Estratégico - Conceitos-Metodologia-Práticas. 34a edição. Grupo GEN, 2018.

QUITÉRIO, F. N. D. Uma análise de técnicas do Planejamento e Controle da Produção e da filosofia Lean. São Carlos, SP: USP, 2010. 98p. (Trabalho de conclusão de Curso de Engenharia de Produção). Universidade de São Paulo, São Paulo.

UNIVERSIDADE FEDERAL DE JUIZ DE FORA. CONGRAD. Resolução $\mathbf{n}^{\circ}$ 69/2008. Cria os Grupos de Educação Tutorial - GET Institucional. Juiz de Fora: Congrad, 2008. Disponível em: https://www2.ufjf.br/prograd//files/2010/03/RES692008.pdf. Acesso em: 16 out. 2020.

REICHHELD, Frederick F. The Ultimate Question: For Unlocking the Door to Good Profits and True Growth. Harvard Business School Press. February 2006.

SLACK, Nigel; BRANDON-JONES, Alistair; JOHNSTON, Robert. Administração da Produção, 8ª edição. Grupo GEN, 2018.

THIOLLENT, Michel. Metodologia da Pesquisa-Ação. São Paulo: Cortez, 1985.

XENOS, Harilaus G.P. Gerenciando a manutenção produtiva. Belo Horizonte: EDG, 1998. TUTORIAL EDUCATION PROGRAM 
Abstract: Faced with a scenario in which continuous training and excellent management are essential for achieving results the members of the Tutorial Education Group of Production Engineering course of the Federal University of Juiz de Fora (UFJF), opted for decentralize the learnings of their course to extend them to other student organizations, and thus contribute to their development. Therefore, the aim of this article is to show how the implementation of three workshops on the themes of production engineering - Layout, Strategic Planning and 5s - positively impacted in a Tutorial Education Program of Electrical Engineering of UFJF. Action research was used as the study methodology due to the direct action of the researchers with the participants and the resolution of the problems.

Keywords: strategic planning, layout, 5S. 